\title{
Crisis Spreading Model of the Shareholding Networks of Listed Companies and Their Main Holders and Their Controllability
}

\author{
Yuanyuan $\mathrm{Ma}$ (D) $^{1}$ and Lingxuan $\mathrm{Li}^{2}$ \\ ${ }^{1}$ School of Economics, Northeastern University at Qinhuangdao, Qinhuangdao 066004, China \\ ${ }^{2}$ School of Control Engineering, Northeastern University at Qinhuangdao, Qinhuangdao 066004, China \\ Correspondence should be addressed to Yuanyuan Ma; mayuanyuan@mail.neu.edu.cn
}

Received 17 June 2018; Revised 30 September 2018; Accepted 15 November 2018; Published 4 December 2018

Guest Editor: Piotr Brodka

Copyright (C) 2018 Yuanyuan Ma and Lingxuan Li. This is an open access article distributed under the Creative Commons Attribution License, which permits unrestricted use, distribution, and reproduction in any medium, provided the original work is properly cited.

\begin{abstract}
Bankruptcy of listed companies or shareholders delisting usually causes the crisis spreading in stock markets. Based on the systematic analysis of the epidemic diseases and rumors spreading on the complex networks, the SIR model is introduced to research the crisis spreading in shareholding networks of listed companies and their main holders on the basis of the data about ownership structure in Chinese Stock Markets. The characteristics of shareholding networks are studied, and the parameters for the SIR model are obtained by empirical approach. Then, the numerical computation method is successfully used to analyze the crisis spreading in the networks when the networks meet random failures or intentional attacks. We find the networks have good robustness against the random failures. However, the crisis will spread at a high speed and cause catastrophic damage if there are some failures or attacks on hub vertices in the networks. Under this condition, the networks show obvious vulnerability. Last but not least, the controllability of the networks under the condition of intentional attacks and random failures is studied. The results show that if the network is controlled globally, it is more reliable to allow a politically good new or an appropriate exciting economical policy to play the role in orienting markets under the control of public opinions as the crisis occurs. However, under normal circumstances, controlling a small part of driver vertices representing listed companies, applying appropriate control strategies, and using its characteristics of high efficiency of sending information can effectively control the stock market. Our research provides a new reference to further exploration about the transmission mechanism of the crisis based SIR model and further research on the controllability of crisis spreading in financial markets.
\end{abstract}

\section{Introduction}

Reviewing the long course of human history, each financial crisis has led to economic disaster. The Great Depression which started about 1929 and lasted until the late 1930s or early 1940s swept through all the countries in Western Europe and the United States. When the members of Organization of Arab Petroleum Exporting Countries proclaimed an oil embargo against the United States along with the fourth Arab-Israeli War breaking out, the 1973 oil crisis started, which subsequently led to economic crisis. The Latin American debt crisis occurred in the early 1980s (and for some countries starting in the 1970s) known as the "lost decade". Around 1990s, the Japanese asset price bubble collapsed because of the great inflation of real estate and stock prices.
The Asian financial crisis gripped most area in Asia from 1997 to 1998

With Chinese joining in the WTO, our financial market opened to the outside world further. The relationship between Chinese mainland financial system and foreign financial systems has been getting closer. The Chinese stock markets can be influenced by various kinds of crises from aboard. For example, the US subprime mortgage crisis in 2007 triggered the worldwide financial crisis. The closing price of Shanghai securities composite index (index code: 000001 ) falls from the maximum point $6082.06 \mathrm{CNY} /$ point on October 16, 2007 to 1706.7 CNY/point on November 4, 2008. The closing price of the Shenzhen composite index (index code: 399106 ) falls from the maximum point $1576.5 \mathrm{CNY} /$ point On January 15, 2008 to $456.97 \mathrm{CNY} /$ point on November 4, 2008. The 
samples of the index 000001 and index 399106 include all the issued shares in Shanghai security exchange and Shenzhen security exchange, respectively. It means that more than $70 \%$ Market Capitalization had vaporized during the year of 2008.

Invariably, each financial crisis will do very serious damage to the country's real economy. If we can completely master the transmission mechanism of financial crisis and exactly predict the financial crisis, it is possible for government to take steps to nip the crisis in the bud.

It is well known that the stock markets are the barometer of national economic development. The stock prices of listed companies can reflect the capital demand and supply situation, market demand, current situation, anticipation of industry development trend, and the unrest of political situations. The stock markets are so sensitive to the economic crisis and financial crisis that once some abnormal phenomena occur in the stock market, the real economy of the country will be affected inevitably and seriously. It is easy for the abnormality in stock market to trigger a global financial crisis or economic crisis. On the contrary, if some abnormal phenomena occur in the real economy, the bubble in the stock markets will collapse firstly. Then the crisis will penetrate into every aspect of people's lives rapidly. Therefore, studying the structural characteristics of stock markets and the crisis spreading in the stock markets are important.

Along with the study of the transmission mechanism and the statistical mechanics of complex networks, two farreaching spreading models are formed. They are susceptibleinfected-susceptible (SIS) mode [1-4] and SIR model [1, 57]. When talking about SIS and SIR model on networks, the literature typically refers to epidemiological dynamical processes which have been studied for quite a long time. As for the so-called SIR model, it means that each vertex in networks lies in one of the following states: susceptible (healthy state), infected, removed (or refractory, or recovered). At each step, the susceptible vertices become infected vertices with certain probability if they enter in contact with infective vertices and disease transmission occurs successfully. At the same time, the infected vertices become removed (or refractory, or recovered) vertices with certain probability [1,5-7]. Later, the SIR and SIS model are also introduced to describe the rumor spreading process in interpersonal networks since they have the similar spreading process of epidemiology.

Bankruptcy of listed companies or shareholders delisting usually causes the crisis spreading in stock markets. Based on the systematic analysis of the epidemic diseases and rumor spreading on the complex networks, the SIR model will be introduced to research the crisis spreading in shareholding networks of listed companies and their main holders.

Compared with the SIR model of epidemic spreading or rumor spreading on general complex networks, the susceptible-infected-removed (SIR) model of crisis spreading shows great differences in the shareholding networks due to stock markets having their own characteristics. The networks are established by the real data of the mutual investment relationships between the listed companies and their main holders, and the shareholding networks are the typically weighted and directed networks. In order to reduce the loss, the vertices representing the listed companies or the main holders make different decisions according to their different situations. Meanwhile, the decisions made by the listed companies or the main holders may be obviously different. Therefore, the susceptible or infected vertices in the correlated networks will become infected vertices or removed vertices with no certain given probability, respectively. Not only is the SIR model beneficial to master the transmission mechanism of crisis spreading on the stock markets, but also it can help to reveal the dissemination process and root cause of financial crisis.

Compared to model research of crisis spreading, perhaps reflecting the controllability and control strategies for the crisis are more concerned. The ultimate goal of studying complex network systems is controlling, or manual intervention. It involves issues such as controllability, control strategies, precise control, minimum cost control, and spontaneous controllability. In 2011, Liu and the control theory community J. J Slotine and the complex network leader A. L. Barabasi cooperated to use the linear system structure controllability theory and introduced mapping maximum matching for networks and Kalman's controllability rank condition to establish the theory for analyzing the controllability of complex networks [8]. On the basis of Liu's work, Jia et al. divide the network vertices into three categories, critical vertices, intermittent vertices, and redundant vertices, and further calculate the proportion of the three types of driver vertices and propose the concept of control capacity $[9,10]$. Subsequently, Yuan et al. propose a more accurate concept of networks controllability based on the PBH rank criterion and further introduced it into the research of multirelational networks and multilayer networks [11]. Sun et al. consider that, in the control design process of practical complex systems, the control problem of the system is usually considered based on the energy optimal control, which involves the calculation of the controllable matrix, and thus controllability theory based on the singularity of Gramian matrix is proposed [12]. Based on these three types of controllability research, many researchers have carried out various kinds of research.

In this paper, we will not only carry on model research of crisis spreading by establishing a SIR model of crisis spreading in stock markets, but also research the controllability and controlling strategies of shareholding networks when the networks meet random failures or intentional attacks.

This paper can be divided into 7 sections. Section 1 is the introduction about the related subjects and current research. The data and the methods of establishing networks in this paper are elaborated in Section 2. In Section 3, based on the analysis of crisis spreading in the shareholding networks, the SIR model in stock markets is established. Section 4 introduces the characteristics of shareholding networks and the parameters selection for the SIR model. Based on SIR model, Section 5 simulates the process of crisis spreading when the networks meet random failures and intentional attacks. And its controllability of networks has been studied in Section 6 . The main conclusions and some related discussions are given in Sections 7 and 8. 


\section{Data and Networks}

2.1. Data. The data are extracted from the RESSET Financial Research Database (http://www.resset.cn/), including all the stocks issued in the Shanghai Stock Exchange and the Shenzhen Stock Exchange before December 31, 2009. The documents we selected include the Main Stockholders List and Ownership Structure and the Yearly Market Capitalization $(\mathrm{CNY})$ of all listed companies.

Through the issuance of stocks, the shareholding networks between the listed companies and their shareholders are formed. The shareholders of a listed company may be other listed companies, funds, non-listed enterprises, individuals, universities, etc. For each stock, the number of the shareholders in stock markets is numerous. The shareholders holding less proportion stocks may buy or sell their stocks frequently in each trading day. Thus, the shareholders' information constantly changes during exchange hours. To handle all the information of the listed companies and their holders is incredible. However, it is worth noting that the proportion of stocks owned by the major holders of each listed company is more than 55\%. These data can be accessed from the Internet or related financial database. According to our statistics, the proportion of average shares of the 10th largest holders of the listed companies is smaller than $0.42 \%$. Comparatively, the holders holding share smaller than $0.42 \%$ have little influence on the analysis of the community structures and topological characteristics of complex networks.

There is no uniformity in naming convention about the holders' name for the annual reports in the Securities and Futures Commission (SFC), such as full name or abbreviated name, name in Chinese or in English, subsidiary company's name or parent company's name, etc. Therefore, the name of the same holder must be unified. The names of all domestic companies are unified in Chinese. The names of all foreign companies are unified in English. If some different holders are the subsidiary companies of a certain company, we view them as the same vertex in the networks. For example, China Life Insurance Company Limited and China Life Asset Management Company Limited are the subsidiary companies of China Life Insurance (Group) Company. Under this condition, the three companies should be viewed as the same vertex representing their parent company (China Life Insurance (Group) Company).

2.2. Networks. In shareholding networks, the listed companies and their main holders are the vertices, which is different to [13]. The shareholding relationships are the edges of the networks. According to the graph theory [14], the networks can be indicated by the directed graph $G=$ $(V, E)$, where $V$ is a set of vertices, which represents the listed companies and their main holders. $E$ is the ordered pair of vertices, called directed edges, which represents the investment relationships.

For the purpose of indicating the mutual investment relationships in stock markets, the basic subgraph of shareholding networks is extracted, as shown in Figure 1.

In Figure 1, the symbol of hexagons denotes a listed company; the symbol of circles denotes a main holder except

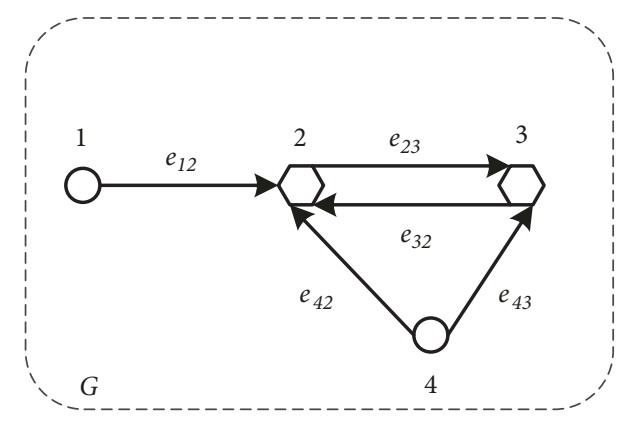

Figure 1: A subgraph of shareholding networks.

the listed companies (in this paper, denoted as nonlisted holders). The set $V$ includes two kinds of vertices: the set of listed companies (denoted by $V_{L}$ ) and the set of main holders (denoted by $V_{S}$ ), where $V_{L} \subset V, V_{S} \subseteq V$. It is worth noting that some listed companies can also act as the main holders of some other listed companies, such as Company 2 and Company 3 as shown in Figure 1. Thus, we have $v_{2}, v_{3} \in V_{L}$ and $v_{1}, v_{2}, v_{3}, v_{4} \in V_{S}$.

The set $E$ indicates the mutual investment relationships between the listed companies and their main holders. If holder $i$ holds certain proportion of stocks issued by the listed company $j$, holder $i$ has invested in the listed company $j$. In the shareholding networks, the investment relationships can be represented by the ordered pair $e_{i j}=\left(v_{i}, v_{j}\right)$; the direction is from vertex $v_{i}$ to vertex $v_{j}$. Meanwhile, the weight between vertex $v_{i}$ and vertex $v_{j}$ is denoted as the symbol $u_{i j} . u_{i j}$ is the proportion of the holding shares of holder $i$ to the total issued shares by the listed company $j . u_{i j}=0$ indicates that there are no investment relationships between the holder $i$ and the listed company $j$. If $e_{23} \neq 0$ and $e_{32} \neq 0$, $e_{23}=\left(v_{2}, v_{3}\right)$ and $e_{32}=\left(v_{3}, v_{2}\right)$ will represent the difference investment relationships in the shareholding networks. In order to indicate the possible existing investment relationships in the shareholding networks, all kinds of shareholding relationships are listed in Table 1 on the basis of analyzing Figure 1.

The Yearly Market Capitalization of any listed company is the price of stocks issued by the listed company multiplied by the total issued shares. Suppose $M_{j}$ as the Yearly Market Capitalization of the listed company $j$. The asset of holder $i$ investing to listed company $j$ is $u_{i j}$ multiplied by $M_{j}$. Now, we can define the in-degree assets of listed company $j$ as investment of their holders. We use $s_{j}^{\text {in }}$ to represent the indegree assets of listed company $j$, then

$$
\begin{aligned}
s_{j}^{i n} & =\left(u_{1 j}+u_{2 j}+\cdots+u_{i j}+\cdots+u_{N_{L} j}\right) \times M_{j} \\
& =\sum_{i=1}^{N_{L}} u_{i j} \times M_{j}
\end{aligned}
$$

where $N_{L}$ is the total number of the listed companies. By the way, the nonlisted holders have not the in-degree assets. 


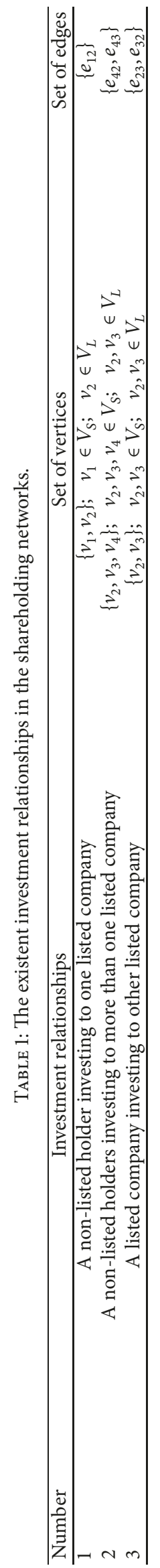


Let the out-degree of nonlisted holder (or listed company) $i$ as its investment to the other listed companies; we use $s_{i}^{\text {out }}$ to represent the nonlisted holder (or listed company) $i$; then

$$
\begin{aligned}
s_{i}^{\text {out }}= & u_{i 1} \times M_{1}+u_{i 2} \times M_{2}+\cdots+u_{i j} \times M_{j}+\cdots \\
& +u_{i N_{s}} \times M_{N_{L}}=\sum_{j=1}^{N_{L}}\left(u_{i j} \times M_{j}\right)
\end{aligned}
$$

\section{Analysis of Crisis Spreading in Shareholding Networks and the SIR Model}

3.1. Susceptible State, Infected State, and Removed State in Shareholding Networks. As mentioned above, SIR models are mainly used to investigate the epidemiological dynamical processes or the rumor spreading processes in social networks. The similar modeling method is introduced to research the crisis spreading processes on the basis of the networks of listed companies and their main holders. Thus, the definitions of the vertices and edges of the networks should be carefully illustrated. The vertices represent the listed companies and the main holders. The objective existent shareholding relationships are the edges of the networks. Thus, the susceptible state, infected state, and removed state in the shareholding networks can be defined as follows.

Susceptible State. For the vertex representing nonlisted holders, it means that the external investment of the vertex has not changed. In other words, the out-degree assets of vertex and the directed edges from vertex to other vertices have not changed. For the vertex representing listed companies, it means that the Market Capitalization of the vertex has not decreased.

Infected State. For the vertex representing nonlisted holders, it means that the out-degree assets of the vertex have decreased; meanwhile, the directed edges from vertex to other vertices may change. For the vertex representing listed companies, it means that the Market Capitalization of the vertex has decreased.

Removed State. For the vertex representing nonlisted holders or listed companies, it means that the vertices are removed from the networks.

It is noteworthy that the susceptible vertices may become infected vertices or removed vertices directly when the states of vertices have changed in the shareholding networks. For example, the holder $i$ only holds the stocks issued by a certain listed company $j$. When the Market Capitalization of company $j$ descend because of mismanagement in business, the state of the vertex $v_{i}$ will become susceptible state if the listed company $j$ is still allowed to be listed on the stock markets. However, the state of the vertices $v_{i}$ and $v_{j}$ will become the removed state if the company goes bankrupt.

In the next sections, each kind of the crisis spreading in shareholding networks is analyzed carefully, and the corresponding functions are obtained. To help the reader understand, this article will explain the corresponding change rules of vertices among susceptible state, infected state, and removed state in detail on the basis of the typical network in Figure 1.

3.2. The Existent Failures in Networks. According to graph theory [14], the existent failures in networks can be divided into two categories.

3.2.1. Cut Edges. Suppose $e$ is an edge in graph $G$, and a cut edge $e$ of graph $\mathrm{G}$ means deleting the edge $e$ from graph G; it can be denoted by $G-e$. If $T=\left\{e_{1}, e_{2}, \cdots, e_{i},\right\}$ is an edge subset of $E$ in graph $G=(V, E)$, deleting the edge subset $T$ from subgraph $G$ can be denoted by $G-T$.

3.2.2. Cut Vertices. A cut vertex $v_{i}$ of graph $\mathrm{G}$ means deleting the vertex $v_{i}$ together with the related incident edges, and the graph will be denoted by $G-v_{i}$. Correspondingly, a cut vertex set $C=\left\{v_{1}, v_{2}, \cdots, v_{i}\right\}$ of graph $\mathrm{G}$ means deleting the vertices $C=\left\{v_{1}, v_{2}, \cdots, v_{i}\right\}$ together with the related incident edges, and the subgraph will be denoted by $G-C$.

3.3. Crisis Spreading Model of SIR in Shareholding Networks. Compared to the SIR model of epidemic spreading or rumor spreading in complex networks, the SIR model of crisis spreading shows no given susceptible or infected probabilities in shareholding networks, in which the crisis spreading between any two vertices is influenced by many factors, such as shareholding proportion, Market Capitalization, in-degree assets, and out-degree assets. Meanwhile, the crisis spreading has obvious directions in the shareholding networks because the networks are established by the real data of the mutual investment relationships between the listed companies and their main holders.

After careful analysis, the failure in stock markets can be divided into 5 categories. Correspondingly, the crisis spreading functions in shareholding networks can be obtained as follows.

3.3.1. Influence of Nonlisted Holders' Bankruptcy. Suppose nonlisted holder $i$ goes bankrupt; in shareholding networks the vertex $v_{i}$ should be deleted; meanwhile, the edges linking vertex $v_{i}$ to other vertices should also be deleted.

According to the proportion $u_{i j}$ of the holding shares of holder $i$ to the total issued shares by listed company $j$, the listed company $j$ may be influenced. This kind of influence can be denoted by equation $C_{2}\left(v_{i}, v_{j}, u_{i j}\right)$. Then, the bankruptcy of nonlisted holder $i$ can be described as in

$$
H_{1}\left(v_{i}\right)=\left\{\begin{array}{l}
G-v_{i} \\
C_{2}\left(v_{i}, v_{j}, u_{i j}\right)
\end{array}\right.
$$

where $j=1,2,3, \cdots, n$, and $v_{j} \in V_{L} ; v_{i} \in V_{S}, v_{j} \notin V_{L} . n$ is the total number of vertices before we delete the vertex $v_{i}$. If there is no investment relationship between the nonlisted holder $i$ and listed company $j, u_{i j}=0$. Under this condition, let $C_{2}\left(v_{i}, v_{j}, u_{i j}\right)=0$. That is to say, the nonlisted holder $i$ which goes bankrupt has no direct influence on listed company $j$.

In order to improve the replicability of this paper and master the functions and parameters for casual readers, five figures and many paragraphs are added to illustrate the rules 


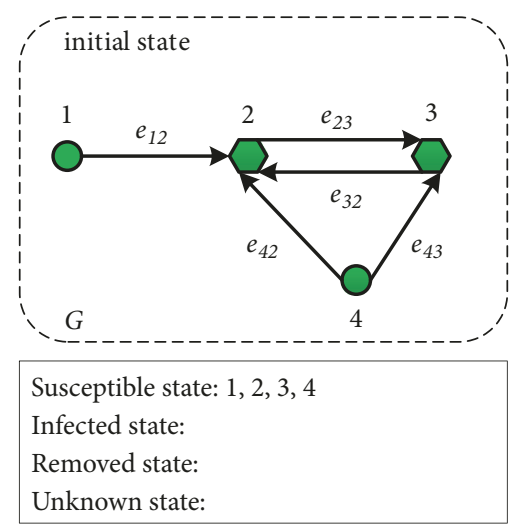

(a) Initial state

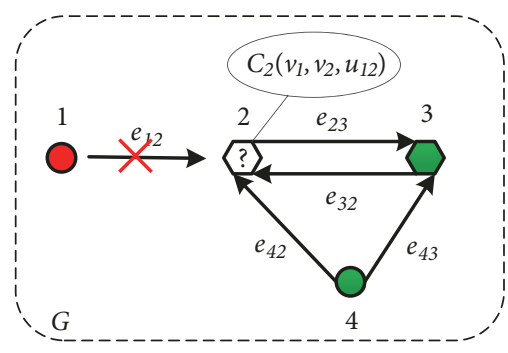

Susceptible state: 3,4

Infected state:

Removed state: 1

Unknown state: 2

FIGURE 2: Influence of bankruptcy of nonlisted holder 1 on the network.

of crisis spreading in Section 3.3 (Sections 3.3.1 to 3.3.5) based on simple network of Figure 1. The influence of the bankruptcy nonlisted holder 1 on the network is expressed in Figure 2.

Figure 2 shows that the bankruptcy of nonlisted holder 1 deletes the vertex $v_{1}$ together with the incident edge $e_{12}$; correspondingly, the susceptible state of vertex 1 becomes removed state directly; it also affects the investment relationship of company 2 linked to holder 1, which is denoted by $C_{2}\left(v_{1}, v_{2}, u_{12}\right)$.

3.3.2. Bankruptcy of Listed Companies. When listed company $j$ goes bankrupt, the vertex $v_{j}$ and the edges linking vertex $v_{j}$ to other vertices will be deleted inevitably in the shareholding networks. At the same time, the vertices linked to vertex $v_{j}$ will be influenced.

On the one hand, if listed company $j$ holds the stocks issued by another listed company $k$, the influence of bankrupt company $j$ on listed company $k$ is $C_{2}\left(v_{j}, v_{k}, u_{j k}\right)$, where $k=$ $1,2,3, \cdots, n, k \neq j$.

On the other hand, the total assets of the holders of listed company $j$ will decrease inevitably. The loss assets of holder $i$, who has invested to listed company $j$, can be obtained as follows:

$$
m_{i}=M_{j} \times u_{i j}
$$

where $M_{j}$ is the Market Capitalization of listed company $j$ before going bankrupt.

At this time, holder $i$ will make a decision for benefiting itself according to its situation of loss, which can be described by equation $H_{2}\left(v_{i}, m_{i}\right)$, where $i=1,2,3, \cdots, n$ and $v_{i} \in V_{S}$.

As the analysis above, the changes of the shareholding networks can be described as (5) when the listed company $j$ goes bankrupt.

$$
\begin{aligned}
& C_{1}\left(v_{j}\right) \\
& \quad= \begin{cases}G-v_{j} & i=1,2,3, \cdots, n \& i \neq j \\
H_{2}\left(v_{i}, m_{i}\right) & j=1,2,3, \cdots, n \& k \neq j \\
C_{2}\left(v_{j}, v_{k}, u_{j k}\right) & \end{cases}
\end{aligned}
$$

where $v_{i} \in V_{S}$ and $v_{j}, v_{k} \in V_{L}$.
Using Figure 1 as an example, the influence of the bankruptcy of listed company 2 is shown in Figure 3.

The bankruptcy of listed company 2 makes the edges linking vertex $v_{2}$ to other vertices be deleted inevitably, including $e_{12}, e_{32}, e_{42}, e_{23}$, and the susceptible state of nonlisted holder 1 becomes a removed state because holder 1 only holds the stocks issued by listed company 2 and affects decision behavior of nonlisted holder 4 and listed company 3 because of their assets decreasing, marked by $\mathrm{H}_{2}\left(v_{2}, m_{4}\right)$ and $C_{2}\left(v_{2}, v_{3}, u_{23}\right)$, respectively.

3.3.3. Analysis of the Decision Behavior of Holders When Their Assets Decrease. Under the following two conditions, the assets of holder $i$ will decrease inevitably. (1) Holder $i$ holds more than one kind of stocks issued by different listed companies. The total assets of the holder will decrease inevitably when one of the listed companies goes bankruptcy. (2) If holder $i$ holds the stocks issued by listed company $j$, the assets of holder $i$ will also be decreased inevitably when the Market Capitalization of listed company $j$ descends because of mismanagement in business or some other reasons. Under these two conditions, the holder $i$ will make a decision for benefiting itself, so that it can decrease the losses to a tolerant level.

Suppose $m$ is the loss of assets of holder $i$ before making the decision ( $m$ can be obtained from (6) and (10) for the two conditions mentioned above, respectively.). The holder $i$ will make the decision according to the proportion of the loss to the total external investing assets of holder $i$ (denoted as $r_{i}$ ). If $M_{j}$ is the Market Capitalization of listed company $j, r_{i}$ can be obtained from the following equation:

$$
r_{i}=\frac{m_{i}}{m_{i}+\sum_{\substack{j=1 \\ i \neq j}}^{n}\left(u_{i j} \times M_{j}\right)}
$$

where $v_{i} \in V_{S}, v_{j} \in V_{L}, j \neq i . n$ is the total number of the listed companies. If holder $i$ does not invest in the listed company $j, u_{i j}=0$.

According the value of $r_{i}$, the loss of holder $i$ can be divided into 3 kinds: general loss, heavy loss, and catastrophic 


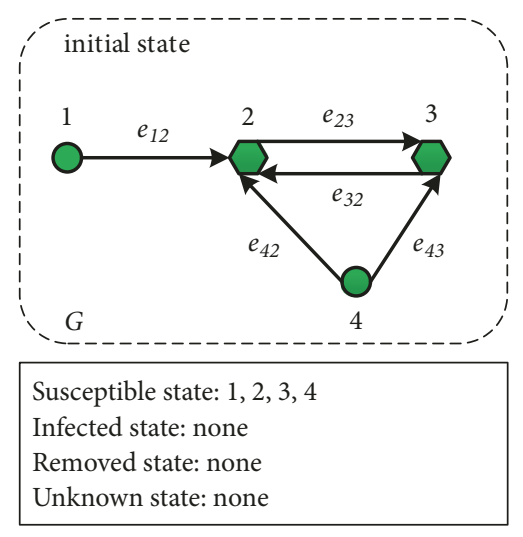

(a) Initial state

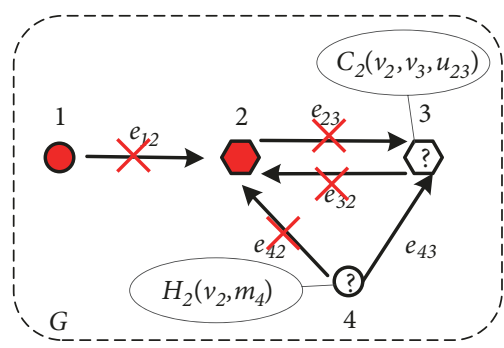

Susceptible state: Infected state:

Removed state: 1,2

Unknown state: 3,4

(b) Result of iinfluence

FIGURE 3: Influence of bankruptcy of listed company 2 on the network.

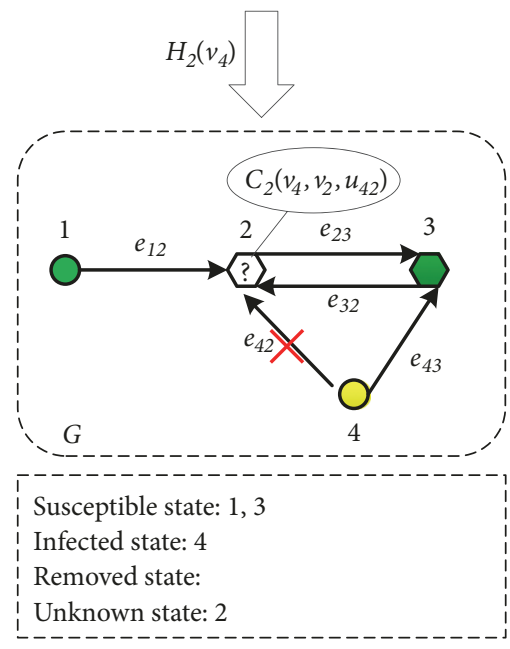

(a) Heavy loss of holder 4 (vertex 4$)$

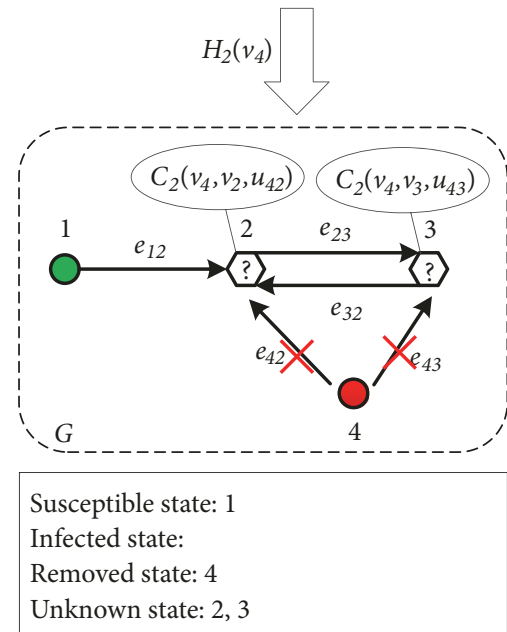

(b) Catastrophic loss of holder 4 (vertex 4$)$

Figure 4: Decision behavior of holder 4 when it suffers a heavy or catastrophic loss.

loss. Correspondently, the decision behavior of holder $i$ can be described by the following equation:

$$
\begin{aligned}
& H_{2}\left(v_{i}, m_{i}\right) \\
& \quad= \begin{cases}0 & 0 \leq r_{i}<x_{1} \\
G-e_{i j} \text { and } C_{2}\left(v_{i}, v_{j}, u_{i j}\right) & x_{1} \leq r_{i}<x_{2} \\
H_{1}\left(v_{i}\right) & r_{i} \geq x_{2}\end{cases}
\end{aligned}
$$

where $i \neq j, v_{i} \in V_{S}$ and $v_{j} \in V_{L} \cdot x_{1}$ and $x_{2}$ can be obtained from Section 4.2.

Equation (7) can be described as follows.

If $r_{i}<x_{1}$ (general loss), the holder $i$ will make no new decision. Correspondently, the vertex $v_{i}$ will keep the same state as before in the shareholding networks.

If $x_{1} \leq r_{i}<x_{2}$ (heavy loss), holder $i$ will sell one kind of stocks issued by company $j$. The assets of holder $i$ investing in listed company $j$ are minimum (not including zero) compared to its investment to other companies. Correspondently, in the networks, the edge $e_{i j}$ will be deleted and the influence of holder $i$ selling the stocks issued by company $j$ on company $j$ can also be described by equation $C_{2}\left(v_{i}, v_{j}, u_{i j}\right)$.

If $r_{i} \geq x_{2}$ (catastrophic loss), the holder $i$ will sell all the holding stocks. It can be described by equation $H_{1}\left(v_{i}\right)$.

For the last two conditions, they can be mastered by the example in Figure 4.

The initial state of the networks is the same as Figure 3(a). Assuming the assets of holder 4 investing in company 2 are smaller than those in company 3 , when holder 4 suffers a heavy loss, it will sell the stocks issued by company 2; correspondingly, the influence on company 2 expressed by $C_{2}\left(v_{4}, v_{2}, u_{42}\right)$ and the susceptible state of vertex 4 becomes an infected state, as shown in Figure 4(a). When holder 4 gets a catastrophic loss, it will sell all the holding stocks and influence on the listed companies, as denoted by $C_{2}\left(v_{4}, v_{2}, u_{42}\right)$ and $C_{2}\left(v_{4}, v_{3}, u_{43}\right)$ in Figure $4(\mathrm{~b})$. 
3.3.4. Influence of Holder $i$ on the Networks When Holder $i$ Goes Bankrupt or Sells the Stocks Issued by Listed Company $j$. When holder $i$ goes bankrupt or sells the stocks issued by listed company $j$, the Market Capitalization of listed company $j$ may be influenced. Suppose $M_{j}$ is the Market Capitalization before holder $i$ goes bankrupt or sells the stocks issued by listed company $j$ and $M_{j}^{\prime}$ is the new Market Capitalization after holder $i$ goes bankrupt or sells the stocks issued by listed company $j$. Suppose $R_{j}$ is the proportion of $M_{j}^{\prime}$ to $M_{j}$. Then,

$$
R_{j}=\frac{M_{j}^{\prime}}{M_{j}}
$$

According to the Chinese Stock Exchange Listing Rules, the behaviors of selling or buying assets of the holders who hold the proportion of shares more than $5 \%$ of a certain listed company are viewed as major events in stock markets. The behaviors of the holder, who holds the proportion of shares more than $50 \%$ of a certain listed company, selling or buying assets are viewed as the behaviors of the listed company [15]. Therefore, we neglect the influence, which the proportion of single transaction shares of a certain listed company to its total shares is less than $5 \%$. After careful analysis of the block trade of stocks in the stock markets, the new Market Capitalization $M_{j}^{\prime}$ is obtained, as shown in (12).

Equation (8) shows $R_{j} \geq 0$. Moreover, if $R_{j}$ is more closer to 1 , the fluctuation of the Market Capitalization of listed company $j$ is smaller, so the damage to company $j$ is smaller. When $R_{j}$ is a certain value $x_{3}$, the listed company $j$ will go bankrupt. The value of $x_{3}$ can be found in Section 4.2.

Therefore, when holder $i$ goes bankrupt or sells the stocks issued by listed company $j$, the influence of holder $i$ on listed company $j$ can be written as in the following equation:

$$
\begin{aligned}
& C_{2}\left(v_{i}, v_{j}, u_{i j}\right) \\
& = \begin{cases}0 & u_{i j}<0.05 \\
C_{1}\left(v_{j}\right) & u_{i j} \geq 0.05 \text { and } 0 \leq R_{j} \leq x_{3} \\
C_{3}\left(v_{j}\right) \text { and } H_{2}\left(v_{\mathrm{q}}, m_{\mathrm{q}}\right) & u_{i j} \geq 0.05 \text { and } 1>R_{j}>x_{3} \\
0 & R_{j} \geq 1\end{cases}
\end{aligned}
$$

where $q=1,2,3, \cdots, n, q \neq j, i \neq j, i \neq \mathrm{q}, v_{i}, v_{q} \in V_{S}, v_{j} \in V_{L}$. The meanings of (9) are as follows.

If $u_{i j}<0.05$, the behaviors of holder $i$ going bankrupt or selling the stocks issued by company $j$ will have no direct influence on company $j$.

If $u_{i j} \geq 0.05$ and $0 \leq R_{j} \leq x_{3}$, the behaviors of holder $i$ going bankrupt or selling the stocks issued by company $j$ will make company $j$ go bankrupt or be delisted from the stock markets.

If $u_{i j} \geq 0.05$ and $1>\mathrm{R}_{j}>x_{3}$, the behaviors of holder $i$ going bankrupt or selling the stocks issued by company $j$ will make the Market Capitalization of company $j$ decrease, but the company $j$ will not go bankrupt. At this time, the company $j$ will make different decisions according to its decrement of Market Capitalization (denoted as $C_{3}\left(v_{j}\right)$, where, $v_{j} \in V_{L}$ ). Meanwhile, the holders of company $j$ will make different decisions according to its assets decrement, which is the same as $H_{2}\left(v_{q}, m_{q}\right)$, where $q=1,2,3, \cdots, n$, $q \neq j, \mathrm{q} \neq i$ and $v_{q} \in V_{S}$.

$R_{j} \geq 1$ means that the Market Capitalization of company $j$ has not decreased. Therefore, the vertex $v_{j}$ will keep the same state as before in the shareholding networks.

By the way, when $1>R_{j}>x_{3}$, the assets of loss of holder $q$ (denoted by $m_{\mathrm{q}}$ in (9)) can be obtained from the following equation:

$$
m_{\mathrm{q}}=\left(M_{j}-M_{j}^{\prime}\right) \times u_{q j}
$$

Using the network in Figure 1 as an example, if Holder 4 sells the stocks issued by company 2 , the state of vertex 4 will be infected state. The corresponding influence on the networks can be depicted in Figure 5.

As shown in Figure 5(b), it will not impact on Company 2 when the proportion $u_{i j}$ is less than 0.05 or Market Capitalization of company 2 has not decreased. However, if the Market Capitalization of company 2 shows a large decrease and $u_{42} \geq 0.05$, company 2 will go bankrupt (see Figure 5(c)). In addition, there is a middle state between Figures 5(b) and 5(c). Figure 5(d) shows that holder 4 selling the stocks issued by company 2 will make the Market Capitalization of company 2 decrease; i.e., the state of vertex 2 becomes infected state. It is worth noticing that company 2 can make a different decision according to its decrease values, marked by $C_{3}\left(v_{2}\right)$. Furthermore, as one of the holders of company 2 , listed company 3 also will make a decision on account of the assets decrease, denoted by $\mathrm{H}_{2}\left(v_{3}, m_{3}\right)$.

\subsubsection{Decision Behavior of Listed Company $j$ When Its Market} Capitalization Decreases. Because the stock prices are the barometer of the economic development, which is sensitive to the general operating conditions, capital supply and demand, market demand, etc., generally speaking, the stock prices rise with the improvement of business performance. Therefore, the highly descent speed of Market Capitalization of listed companies means bad operation conditions. The phenomena of reduced cash flow or the fracture of capital chain may appear. At this time, the listed company may sell the holding stocks issued by some other listed companies to keep the company's normal operating cycle.

As mentioned in (9), if $0 \leq R_{j} \leq x_{3}$, the company $j$ will go bankrupt or be delisted from the stock markets. So company $j$ must take measures to avoid $R_{j}$ approaching to $x_{3}$. Therefore, we can set a critical value $x_{4}$. If $x_{3}<R_{j} \leq x_{4}$, the company $j$ will make the corresponding adjustment to avoid going bankrupt or being delisted from the stock markets.

To sum up, the decision behavior of listed company $j$ can be written as (11) when its Market Capitalization decreases.

$$
\begin{aligned}
& C_{3}\left(v_{j}\right) \\
& = \begin{cases}0 & u_{j k} \equiv 0 \text { or } R_{j}>x_{4} \\
G-e_{j k} \text { and } C_{2}\left(v_{j}, v_{k}, u_{j k}\right) & \exists u_{j k}>0 \text { and } x_{3}<R_{j} \leq x_{4}\end{cases}
\end{aligned}
$$

where $k=1,2,3, \cdots, n$ and $v_{j}, v_{k} \in V_{L}$.

The meanings of (11) are as follows. 


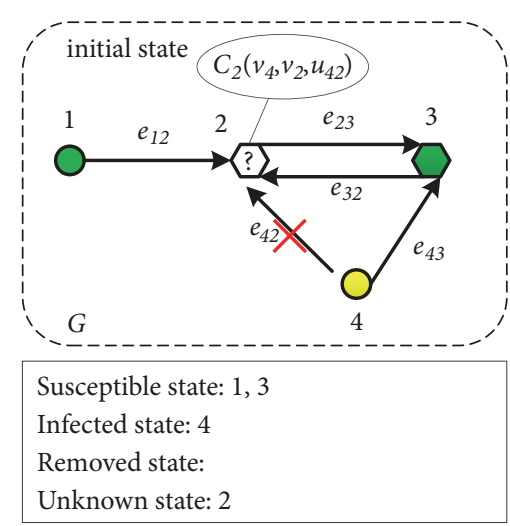

(a) Initial state

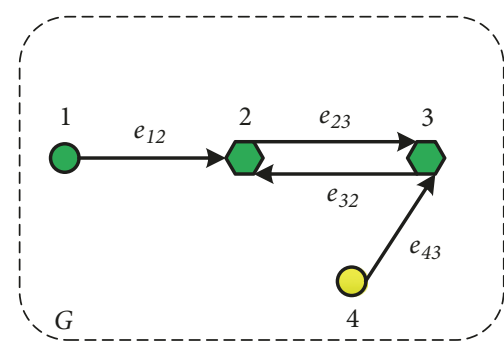

Susceptible state: $1,2,3$

Infected state: 4

Removed state:

Unknown state:

(b) $u_{42}<0.05$ or $R_{2} \geq 1$

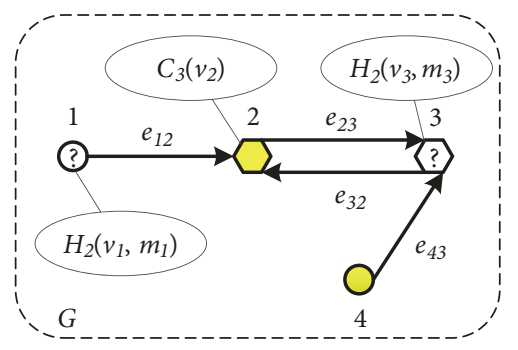

Susceptible state:

Infected state: 2,4

Removed state:

Unknown state: 1,3

(d) $u_{42} \geq 0.05$ and $x_{3}<R_{2}<1$

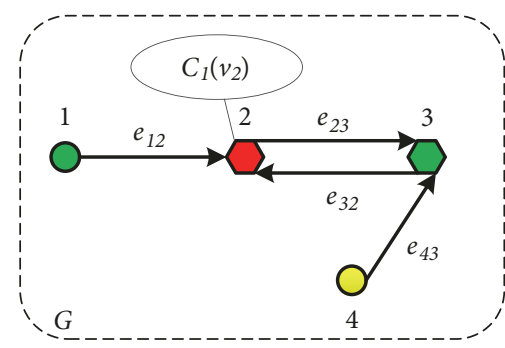

Susceptible state: $1,2,3$

Infected state: 4

Removed state:

Unknown state:

(c) $u_{42} \geq 0.05$ and $0 \leq R_{2} \leq x_{3}$

FIGURE 5: Influence of holder 4 on the networks when holder 4 sells the stocks issued by listed company 2 .

If the listed company $j$ does not hold the stocks issued by some other listed companies $\left(u_{j k} \equiv 0\right)$ or the assets of loss are small $\left(R_{j}>x_{4}\right)$, the company $j$ will make no new decision and the vertex $v_{j}$ keeps the same step as before in the shareholding networks. The vertex $j$ will inherit the last state, such as vertex 2 in Figure 6(a).

If $x_{3}<\mathrm{R}_{j} \leq x_{4}$ and the listed company $j$ holds stocks issued by some other listed companies, the listed company $j$ will sell the stocks issued by company $k$ according to the increase order of the assets which the company $j$ invested to other companies. In the shareholding networks, the edge $e_{j k}$ will be deleted and the influence of company $j$ on listed company $k$ when company $j$ sells the stocks issued by listed company $k$, denoted by $C_{2}\left(v_{j}, v_{k}, u_{j k}\right)$. For example in Figure 1, when the Market Capitalization of company 2 decreases, it will sell the stocks issued by company 3 in order to avoid critical funding shortages, as shown in Figure 6(b).

\section{Characteristics of the Networks and Parameters Selection for the SIR Model}

4.1. Characteristics of the Shareholding Networks. The Market Capitalization of Yearly End Date of the stock markets is $¥ 3.28 \times 10^{13}$ in 2007 and $¥ 1.22 \times 10^{13}$ in 2008 . It denotes the Chinese Stock Markets have an obvious shrinkage phenomenon because of the global financial crisis in 2008, which had a great influence on the Chinese economy. For the purpose of researching the robustness and vulnerability of Chinese stock markets against extreme circumstances, we will choose the data before and after the economic crisis to analyze. The data in 2007 is used as the sample in normal period; the data in 2009 is used as the sample of extreme circumstances after the crisis. As an example to simulate the crisis spreading in the networks, the data on December 31, 2007 , is selected to establish the correlated networks between the listed companies and the main holders. The networks have 1534 listed companies, 13596 vertices, and 19326 edges.

First of all, we should judge the type of the networks. Thus, we did the linear regression analysis between the in-degree assets (or out-degree assets) and the cumulative probability value of the in-degree assets (or out-degree assets) of the vertices of the networks under double logarithmic coordinates. If we use $\gamma$ as the symbol for the slope coefficients obtained by the linear regression analysis of the cumulative distribution, the probability distribution will follow a power law $p(k) \sim k^{-(-\gamma+1)}$ with the exponent $-\gamma+1$. Thus, we use $-\gamma+1$ as the symbol for the scale-free index $[13,16,17]$. Table 2 is the coefficients of linear fit in log-log scale.

The correlated coefficients approach to -1 as shown in Table 2, which indicates that all data points lie on a line 


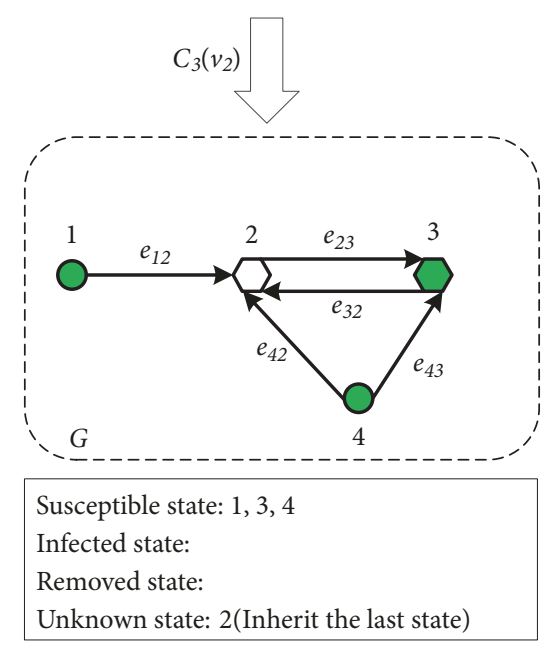

(a) $R_{2}>x_{4}$

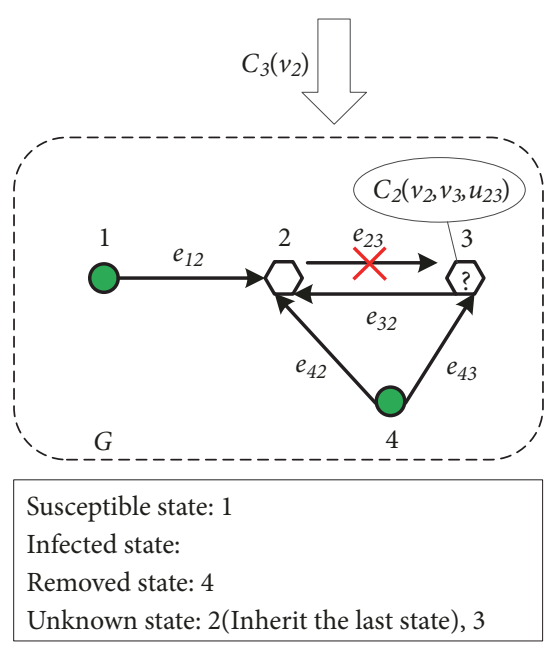

(b) $x_{3}<R_{2} \leqslant x_{4}$

FIGURE 6: Decision behavior of listed company 2 when its Market Capitalization decreases.

TABLE 2: Correlation coefficients and the coefficients of linear fit in log-log scale.

\begin{tabular}{|c|c|c|c|c|c|c|}
\hline \multirow{2}{*}{ Year } & \multicolumn{3}{|c|}{ In-degree assets } & \multicolumn{3}{|c|}{ Out-degree assets } \\
\hline & Correlation & $\gamma^{\text {in }}$ & Constant & Correlation & $\gamma^{\text {out }}$ & Constant \\
\hline 2007 & -0.9272 & -1.3195 & 8.7319 & -0.9655 & -0.4200 & 2.7087 \\
\hline 2008 & -0.9283 & -1.3255 & 8.6392 & -0.9637 & -0.4198 & 2.6787 \\
\hline 2009 & -0.9361 & -1.2552 & 8.7380 & -0.9580 & -0.4214 & 2.7476 \\
\hline
\end{tabular}

for which the cumulative probability value of the in-degree assets (or out-degree assets) decreases as in-degree assets (or out-degree assets) increases in log-log scale. Therefore, the networks belong to the scale-free networks [16-18].

The in-degree assets of the vertices mainly reveal the ability of the listed companies and funds attracting investors. As shown in Table 2, the indices $\gamma^{\text {in }}$ range from -1.2 to 1.4; that is to say, the scale-free indices of in-degree assets of the vertices of the networks are between 2.2 and 2.4. It means that the in-hub vertices can only possess a small part of proportion [16]; i.e., the networks have little super listed companies. The in-hubs mainly represent some large listed companies in Sector I (Finance, Insurance).

The out-degree assets mainly represent the investments from main holders to listed companies and funds. According to Table 2 , the indices $\gamma^{\text {out }}$ range from -0.43 to -0.41 , so the scale-free indices of out-degree assets of the vertices of the networks are between 1.41 and 1.43. Therefore, the distribution of the out-degree assets of the vertices of the networks are also similar to the distribution of the degree of the vertices in sparse scale-free networks. As we know, the mean value and variance of the cumulative distribution function of the degree in sparse scale-free networks are divergent. It means that there are relatively more out-hub vertices in the networks, which mainly represent two kinds of companies: (1) some companies in sector I (Finance, Insurance), such as the state-owned commercial banks and insurance companies and (2) some parent company owning several listed companies. For example, the Aviation Industry
Corporation of China has more than 20 subsidiary companies listing on the stock markets.

\subsection{Parameters Selection for the SIR Model through Empirical} Research. In the RESSET Financial Research Database, the number of stocks with complete information of Market Capitalization of Yearly End Date is 1636 in 2007 and 1804 in 2009. The number of stocks with complete information of Market Capitalization of Yearly End Date both in 2007 and in 2009 is 1628. The Market Capitalization of Yearly End Date of a certain listed company $j$ in 2009 divided by its Market Capitalization of Yearly End Date in 2007 is denoted by $\delta$. Then we get the scatter diagram of cumulative distribution of $\delta$, as shown in Figure 7.

According to Figure 7 , the index $\delta$ ranges from 0.239 to 79.598. Most of the points range from 0.4 to 2.4. The point with maximum value of 79.598 represents Qinghai Salt Lake Industry Group Company Limited (stock code: 000578). By the way, $\delta$ is equal to 79.589 , which is not a normal phenomenon, and the well-known case about salt lake 4.4 billion Yuan equity is always in inquisition stage until now.

On May 8, 2003, Shanghai Stock Exchange and Shenzhen Stock Exchange started a warning mechanism for stocks that is incurring risk of being removed. This is an extra treatment of the Special Treatment Mechanism, and the original stock name will be prefixed with ' $*$ ST'. The daily price up and down limit is also $5 \%$. Furthermore, if the ' $*$ ST' stocks continue to make a loss the next year, they will be temporarily delisted. 


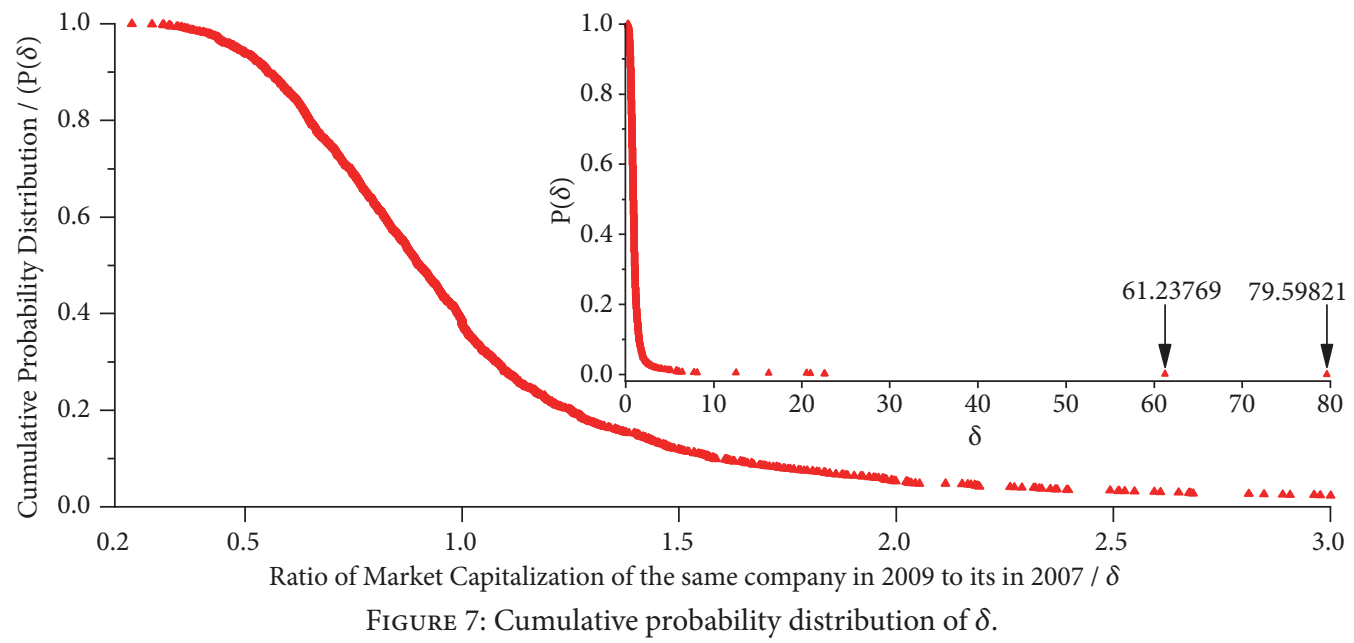

TABLE 3: Fitting parameters of Market Capitalization of Yearly End Date.

\begin{tabular}{lcccc}
\hline \multirow{2}{*}{ Optimization Algorithm } & \multicolumn{2}{c}{$0.05 \leq f<0.5$} & \multicolumn{2}{c}{$0.5 \leq f$} \\
& Differential Evolution & Levenberg-Marquardt & Differential Evolution & Levenberg-Marquardt \\
\hline Mean square error & 0.204586 & 0.204586 & 0.150582 & 0.150582 \\
Root mean squared error & 11.133538 & 11.133545 & 0.453501 & 0.453500 \\
R square & 1.000000 & 1.000000 & 1.000000 & 1.000000 \\
$a$ & 0.959815 & 0.959815 & 0.964046 & 0.964079 \\
$b$ & -0.013473 & -0.013474 & -0.054734 & -0.055387 \\
\hline
\end{tabular}

Checking the original data, we find that 24 listed companies are prefixed with ' $*$ ST'. 8 listed companies are delisted from the stock markets (stock code: 000515, 000569, 420058, 600001, 600357, 600627, 600786, and 600840). The data of Market Capitalization of Yearly End Date in 2009 of them are not found in the RESSET database. We suppose that the delisted companies are the worst operating companies with highly descent speed of Market Capitalization of Yearly End Date. Therefore, $x_{3}$ in (9) is equal to 0.239. Suppose the operating status of the 34 listed companies prefixed with ' $* \mathrm{ST}$ ' is worse than the other listed companies in the stock markets; we can get the critical value $x_{4}$ in (11), which will be equal to 0.419 .

If a holder only holds the stocks issued by one listed company, the holding market value of the holder will become 0.419 times of that in the previous. Under this condition, the best way to decrease the holder's losses is selling the stocks. Therefore, the critical value $x_{2}$ in (7) will be equal to 0.419 .

For $x_{1}$ in (7), Martin Zweig has suggested holders should sell the stocks when its price declines by $10 \% \sim 20 \%$ of Bid Price. So we set $x_{1}=0.2$ in this paper [19].

As mentioned above, we have proved that the probability of the weighted degree of vertices, which represents the Market Capitalization of stocks, followed the power-law. Therefore, we suppose newly Market Capitalization $M_{j}^{\prime}$, previous Market Capitalization $M$, and the proportion of single transaction shares of a certain listed company (denoted by $f$ ) have the following relationships.

$$
\lg M^{\prime}=a \times \lg M+b \times f
$$

where $a, b$ are the parameter.
As mentioned above, the data before and after the Subprime Crisis are selected to analyze the crisis spreading in the networks. Now, suppose the Market Capitalization of Yearly End Date in 2007 is $M$ and in 2009 is $M^{\prime}$. The block trade of stocks with proportion greater than 5\% in RESSET database is used as the sample data. The number of valid sample data sets is 286 . The number of sample data sets with $0.05 \leq f<0.5$ is 266 , and the number of sample data sets with $0.5 \leq f$ is 20 . Two methods have been used to find out $a, b$. The results are listed in Table 3 .

As shown in Table 3, the $\mathrm{R}$ square approaches to 1 even retaining 6 decimal places. It denotes that the block trades of stocks with proportion greater than $5 \%$ can induce the listed companies' Market Capitalization fluctuation. Meanwhile, (12) just can reveal the changes.

According to the analysis shown in Table 3, we set the $\mathrm{a}=$ $0.959815, \mathrm{~b}=-0.013473$ when $0.05 \leq f<0.5$, and $\mathrm{a}=$ $0.964046, \mathrm{~b}=-0.054734$, when $0.5 \leq f$.

\section{Numerical Simulation of Crisis Spreading}

The MATLAB program is used to simulate the crisis spreading in the shareholding networks under the condition of the networks meeting random failure or intentional attack. The random failure refers to removing the vertices randomly. The intentional attack refers that the vertices are removed from big to small according to the degree of the vertices.

The number of initial failure vertices ranges from 0 to 500 . When the number of the initial failure vertices is equal to 500 , it includes 367 listed companies and 133 nonlisted holders under the condition of the networks meeting intentional 


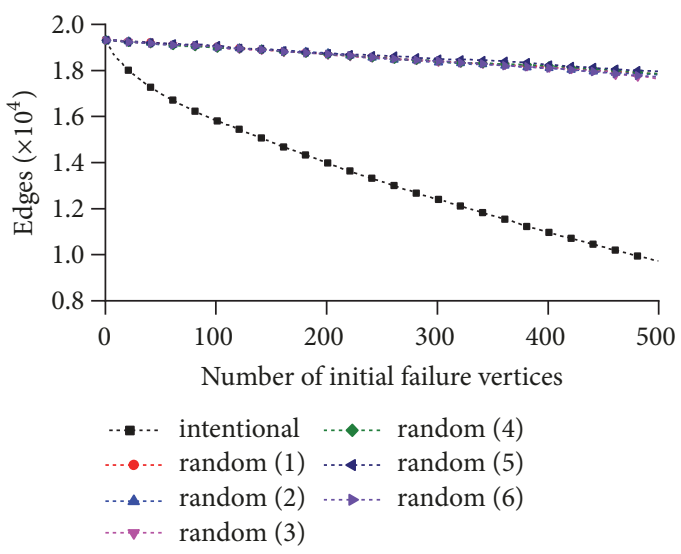

FIgURE 8: Relationships between the edges and the initial failure vertices in the networks.

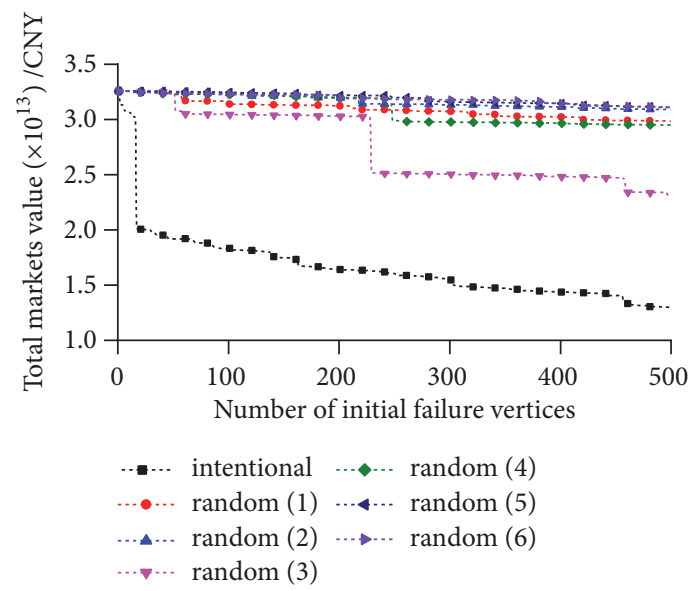

FIGURE 9: Relationships between the total markets value and the initial failure vertices in the networks.

attack. As for the analysis of crisis spreading under the condition of the networks meeting random failure, we select the six sample data, including $48,56,57,51,52$, or 58 listed companies, respectively.

After carefully analyzing, the relationships between the edges and the initial failure vertices in the networks are plotted in Figure 8. The relationships between the total Market Capitalization and the initial failure vertices in the networks are shown in Figure 9.

The random failure can reveal that a handful of listed companies go bankrupt or nonlisted holders are delisted from the stock market under normal circumstances. As shown in Figures 8 and 9, we note that the crisis does not widely spread in the stock markets under this condition. The networks as a whole have good robustness to the random failures. Even with 500 initial failure vertices, the total Market Capitalization of stock markets has declined a little.

The intentional attack can reveal the breakdown of a handful of hub vertices which represents large-scale listed companies and holding companies going bankrupt. Under this condition, the crisis has widely spread in the stock markets and produced quite a lot of damage. There is highly descent speed of total Market Capitalization and the edges of the networks (see Figures 8 and 9). The network has changed obviously and collapsed at a tremendous speed when the number of the initial failure vertices reaches 15 . It denotes that the networks have obvious vulnerability to the intentional attacks. The breakdown of Fannie Mae and Freddie Mac which caused the financial crisis in 2007 is similar to intentional attack in the shareholding networks.

When networks meet random failure and intentional attack, the number of the susceptible state, infected state, and removed state of nonlisted shareholders is listed in Figure 10. The number of the susceptible state, infected state, and removed state of listed companies is listed in Figure 11.

Generally, both Figures 10(a) and 11(a) show that the number of susceptible vertices representing nonlisted holders and listed companies under the condition of the networks meeting random failure is more than that under the condition of the networks meeting intentional attack, respectively. Both Figures $10(\mathrm{c})$ and $11(\mathrm{c})$ show that the number of removed vertices representing nonlisted holders and listed companies under the condition of the networks meeting random failure is less than that under the condition of the networks meeting intentional attack, respectively. These phenomena coincide with the phenomena revealed in Figures 8 and 9; i.e., the networks have good robustness to the random failures and the obviously vulnerability to the intentional attacks. Such characters accompany with the existence of the highly linked hub vertices in scale-free networks, because intentional attack means removing the vertices beginning with the biggest hub vertices and the robustness of the networks can be destroyed easily under the intentional attacks. As mentioned in Ma e Zhuang et al., 2011, and Section 4, the hub vertices mainly represent the super-scale state-owned enterprises, commercial banks, and insurance companies. The closed mutual investment relationships among the hub vertices make them group rich-club spontaneously. There is little linkage between the members of rub-club and the other vertices. Thus, the network shows obviously catastrophic phenomenon. It can just explain why Figures 8 and 9 present a breakpoint.

As proved in Section 4, the networks belong to the scalefree networks in which the linkages among vertices are different. Most of the listed companies have not closely interacted together directly. Thus, the number of listed companies at infected state is small (see Figures 10(b) and 11(b)) either the network meeting random failure or intentional attack. As for the intentional attack on the network, it means that the members of hub-club have been attacked. Under this condition, the network will take on the phenomenon that the same listed companies have been attacked repeatedly because of the closely linkages among the members of hubclub. Therefore, the number of the vertices at infected state is relatively small.

The coordinate axes of Figures 10 and 11 are uniformed by a unified way. Moreover, it is worthy to note that the Yaxis scales (multiplied by $10^{2}$ and $10^{3}$, respectively) are ten times different, respectively. Actually, more than $88 \%$ vertices in the shareholding networks represent nonlisted holders. Therefore, Figure 11 can reveal the overall changing trends of 


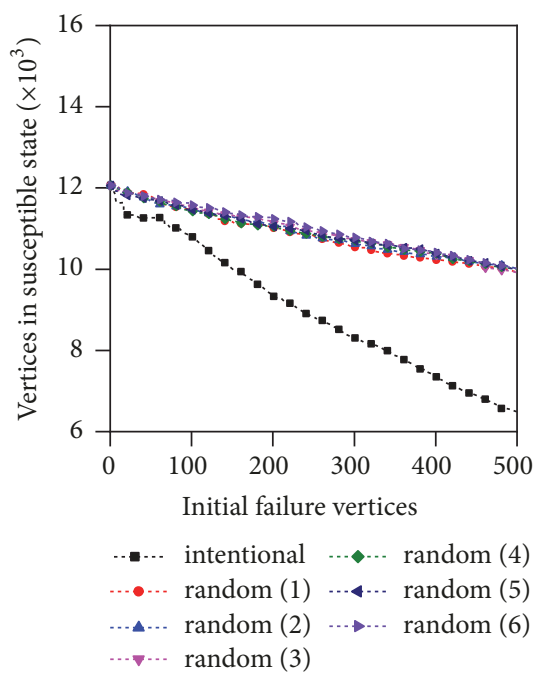

(a)

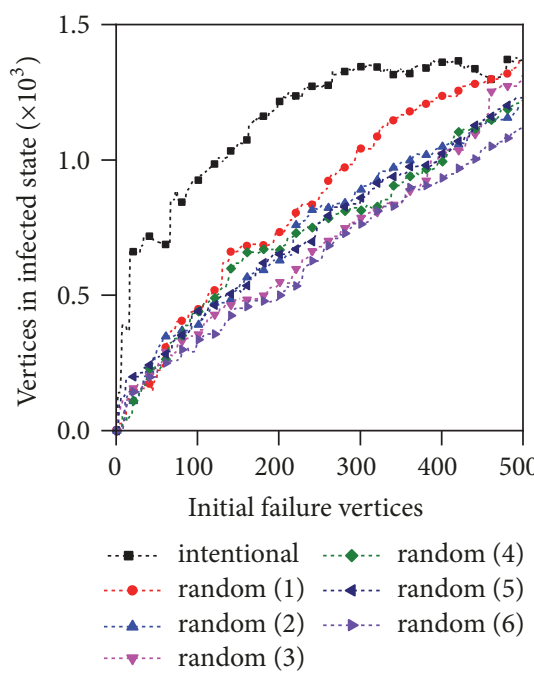

(b)

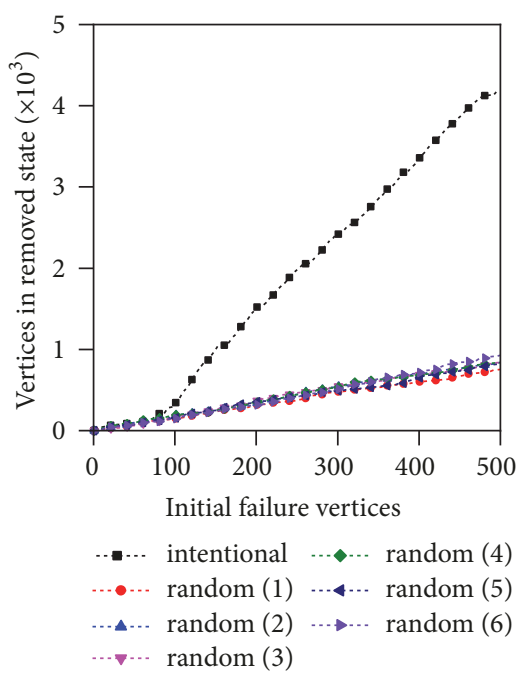

(c)

FIGURE 10: Crisis spreading when networks meet random failure and intentional attack (nonlisted shareholders).

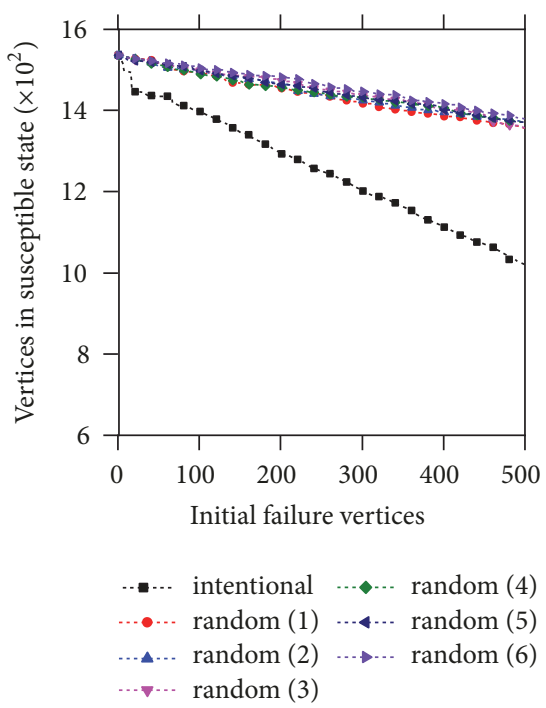

(a)

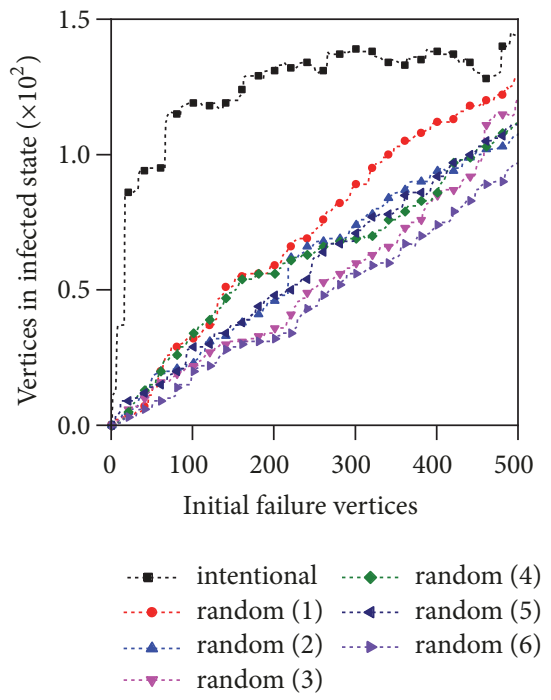

(b)

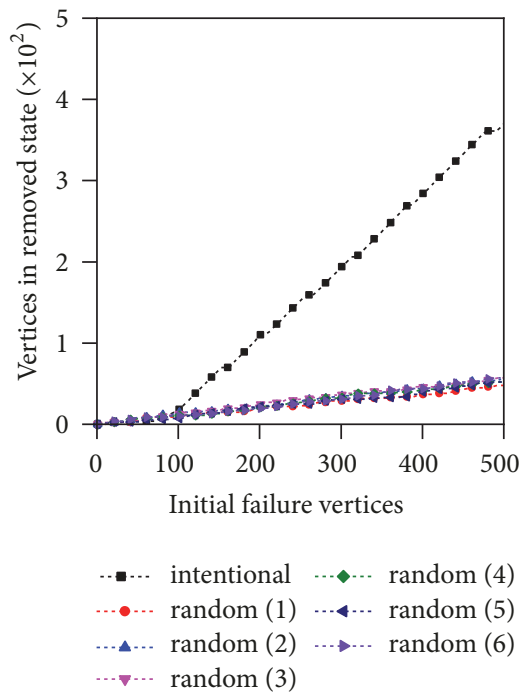

(c)

FIGURE 11: Crisis spreading when networks meet random failure and intentional attack (listed companies).

susceptible vertices, infected vertices, and removed vertices of the networks.

When the number of initial failure vertices is less than 5 , the number of susceptible (infected or removed) vertices representing nonlisted holders and listed companies under the condition of intentional attack is similar to that under the condition of the networks meeting random failure, respectively (see Figures 10 and 11); i.e., a small quantity of hub vertices removing from the networks has not decreased the scale of the networks obviously. Moreover, the crisis has not widely spread in the networks when the number of initial vertices is small. It is mainly because the large-scale listed companies' holders are also handing some other listed companies' stocks. They have good tolerance to the failures in stock markets when we only consider the capital chain between the listed companies and main holders and neglect the rumor spreading in complex networks.

In addition, there is an interesting phenomenon emergence that the fat-tailed degree distribution diverges as the growth number of removed vertices when the networks meet intentional attacks. The scale-free properties are not preserved due to removing the hub vertices continually. Just as Moore C. et al. [20] and Piccardi C. et al. [21] point that the scale-free properties may not preserve in the long run if there is disappearance or death of vertices. 
TABLE 4: Controllability of the shareholding networks under attacks or failures.

\begin{tabular}{|c|c|c|c|c|c|c|c|c|}
\hline \multirow{2}{*}{ Number of initial failure vertices } & \multicolumn{4}{|c|}{ Intentional Attack } & \multicolumn{4}{|c|}{ Random Failure } \\
\hline & $n_{D}$ & $n_{D s}$ & $n_{D c}$ & $n_{D C}$ & $n_{D}$ & $n_{D s}$ & $n_{D c}$ & $n_{D C}$ \\
\hline 0 & 0.8897 & 0.9969 & 0.0031 & 0.0241 & 0.8897 & 0.9969 & 0.0031 & 0.0241 \\
\hline 50 & 0.8892 & 0.9969 & 0.0031 & 0.0242 & 0.8894 & 0.9969 & 0.0031 & 0.0240 \\
\hline 100 & 0.8879 & 0.9970 & 0.0030 & 0.0231 & 0.8891 & 0.9969 & 0.0031 & 0.0245 \\
\hline 150 & 0.8854 & 0.9969 & 0.0031 & 0.0232 & 0.8887 & 0.9969 & 0.0031 & 0.0243 \\
\hline 200 & 0.8837 & 0.9967 & 0.0033 & 0.0245 & 0.8884 & 0.9968 & 0.0032 & 0.0245 \\
\hline 250 & 0.8824 & 0.9964 & 0.0036 & 0.0260 & 0.8880 & 0.9968 & 0.0032 & 0.0245 \\
\hline 300 & 0.8808 & 0.9966 & 0.0034 & 0.0246 & 0.8876 & 0.9968 & 0.0032 & 0.0245 \\
\hline 350 & 0.8795 & 0.9964 & 0.0036 & 0.0254 & 0.8872 & 0.9968 & 0.0032 & 0.0248 \\
\hline 400 & 0.8774 & 0.9963 & 0.0037 & 0.0256 & 0.8869 & 0.9968 & 0.0032 & 0.0247 \\
\hline 450 & 0.8753 & 0.9962 & 0.0038 & 0.0258 & 0.8866 & 0.9968 & 0.0032 & 0.0244 \\
\hline 500 & 0.8741 & 0.9961 & 0.0039 & 0.02668 & 0.8862 & 0.9967 & 0.0033 & 0.0247 \\
\hline
\end{tabular}

\section{Controllability of Crisis Spreading}

As mentioned above, random failures and intentional attacks have striking difference. It has close similarities with dual strategies of targeted vaccinations for controlling the spread of infectious diseases $[22,23]$. For the purpose of discussing the difference of controllability of shareholding networks, we have studied the controllability and controlling strategies of the shareholding networks when the networks meet random failures or intentional attacks on basis of structure controllability theory [8], which is extracted from mapping maximum matching for networks and Kalman's controllability rank condition. The controllability of the networks under the condition of intentional attacks and random failures is listed in Table 4.

In Table $4, n_{D}$ denotes the controllablility of the networks, which is the ratio of the number of minimum number of driver vertices (denoted by $N_{D}$ ) to the total number of the vertices in the network. $n_{D s}$ and $n_{D c}$ are ratio of the number of driver vertices representing nonlisted holders or listed companies to $N_{D}$, respectively. $n_{D C}$ is the number of driver vertiecs representing the listed companies to the total number of verties representing the listed companies.

As shown in Table 4, the proportion of minimum number of driver vertices $n_{D}$ is as high as $87 \%$, including the vertices representing nonlisted holders about $99 \%$. Such a result seems unreasonable, but we think and consider how things happen in this way. We should note that this theory of controllability pays more attention on controllability the whole networks. Of course, to achieve such an effect, the more driver vertices involve in it, the better results networks get. More than 99\% driver vertices belong to nonlisted holders which means that controlling the whole stock markets relies on some input signals which can act on whole stock markets directly or can induce holders decision-making in an indirect way. Maybe a politically good new or an appropriate exciting economical policy can play the key role together with a proper guide of the public opinion when the markets meet failures or attacks.

In addition, it is worthy to note that the proportion of driver vertices representing listed companies is less than
$0.4 \%$, and it is also less than $3 \%$ of the total number of the listed companies in the networks. So, a question worth thinking deeply is how well it works when only controlling the driver vertices representing listed companies.

To illustrate this problem, the concept of global efficiency in complex networks is introduced here. It is an associated concept with the average path length of the network in graph theory. When the distance between two vertices is shorter, the efficiency of transmitting information between them is higher; that is, the efficiency of transmitting information between two vertices is proportional to the reciprocal of the distance between them. The average efficiency of all vertices in the network can reflect the average efficiency of information sent between vertices in the network. The efficiency of the network (indicated by $\eta_{G}$ ) and the average efficiency (indicated by $\eta_{C}$ ) of the listed company in the driver vertices are given in Table 5.

However, under normal circumstances, controlling a small part of driver vertices representing listed companies, applying appropriate control strategies, and using its characteristics of high efficiency of sending information, can effectively control the stock market. Our research provides a new reference to further exploration about the transmission mechanism of the crisis based SIR model and further research on the controllability of crisis spreading in financial markets.

As can be seen from Table 5, the global efficiency of the network is significantly lower. However, it is worth noting that $\eta_{C}$ is not only obviously high, but also gradually reduced under intentional attacks. This phenomenon is not obvious under random failures. In order to clearly illustrate this phenomenon, the ratio of $\eta_{C}$ to $\eta_{G}$ is given in Table 5. This means that intentional attacks obviously cause substantial damage to the hub vertices in the network with listed companies as the core. However, the damage caused by random attacks is not obvious, and in the case of random attacks, the ratio between $\eta_{C}$ and $\eta_{G}$ is more than 1000 times. Combined with Table 4, this clearly reveals that the control of $2 \%-3 \%$ driver vertices representing listed companies, the application of appropriate control strategies, and the use of its high efficiency of sending information can effectively control the trends of stock market to a certain degree in case of random failures. 
TABLE 5: Efficiency of the shareholding networks under attacks or failures.

\begin{tabular}{|c|c|c|c|c|c|c|}
\hline \multirow{2}{*}{ Number of initial failure vertices } & \multicolumn{3}{|c|}{ Intentional Attack } & \multicolumn{3}{|c|}{ Random Failure } \\
\hline & $\eta_{G}$ & $\eta_{C}$ & $\eta_{C} / \eta_{G}$ & $\eta_{G}$ & $\eta_{C}$ & $\eta_{C} / \eta_{G}$ \\
\hline 0 & $2.411 \mathrm{E}-04$ & 0.2908 & 1206.1 & $2.411 \mathrm{E}-04$ & 0.2908 & 1206.1 \\
\hline 50 & $1.982 \mathrm{E}-04$ & 0.1554 & 784.1 & $2.420 \mathrm{E}-04$ & 0.2877 & 1189.0 \\
\hline 100 & $1.902 \mathrm{E}-04$ & 0.1427 & 750.0 & $2.432 \mathrm{E}-04$ & 0.2864 & 1177.6 \\
\hline 150 & $1.993 \mathrm{E}-04$ & 0.1315 & 659.8 & $2.443 \mathrm{E}-04$ & 0.2849 & 1166.0 \\
\hline 200 & $2.044 \mathrm{E}-04$ & 0.1249 & 611.3 & $2.459 \mathrm{E}-04$ & 0.2827 & 1149.8 \\
\hline 250 & $2.096 \mathrm{E}-04$ & 0.1216 & 580.0 & $2.464 \mathrm{E}-04$ & 0.2764 & 1121.6 \\
\hline 300 & $2.134 \mathrm{E}-04$ & 0.1007 & 471.9 & $2.476 \mathrm{E}-04$ & 0.2720 & 1098.5 \\
\hline 350 & $2.198 \mathrm{E}-04$ & 0.0968 & 440.5 & $2.492 \mathrm{E}-04$ & 0.2716 & 1089.5 \\
\hline 400 & $2.293 \mathrm{E}-04$ & 0.0932 & 406.4 & $2.502 \mathrm{E}-04$ & 0.2693 & 1076.2 \\
\hline 450 & $2.420 \mathrm{E}-04$ & 0.0911 & 376.4 & $2.507 \mathrm{E}-04$ & 0.2652 & 1057.5 \\
\hline 500 & $2.465 \mathrm{E}-04$ & 0.0794 & 322.1 & $2.514 \mathrm{E}-04$ & 0.2622 & 1042.7 \\
\hline
\end{tabular}

Just as shown in Table 5, the small part of driver vertices representing listed companies, which mainly denote some large-scale listed companies, has high efficiency of sending information ability. Thus, the stock prices of many other listed companies are highly influenced by the stock price of large-scale listed companies. Meanwhile, as mentioned in [13], the highly linked hub vertices mainly represent the super-scale state-owned enterprises, commercial banks, and insurance companies. These companies are indispensable for our life. In addition, in our networks, the nonrational decisions and some other existing nonlinear factors are neglected. Thus, once the large-scale companies go bankrupt, the rumor among the ordinary shareholders and some other unknown factors will accelerate the spreading of the crisis. The bubble in the stock markets will collapse easily. Then the phenomenon of domino effect may appear. Correspondingly, a financial crisis may take place that the US subprime mortgage crisis in 2007 triggering the worldwide financial crisis is just a good case in point. Therefore, when some largescale listed companies go bankrupt, the government must do its best to avoid the rumor spreading in the stock markets and prevent the catastrophe.

\section{Conclusion}

In this paper, the mutual influences between listed companies and their main holders because of the broken financing chain are studied to reveal the crisis spreading in shareholding networks of listed companies and their main holders. The crisis-spreading model of susceptible-infected-removed (SIR) is established. The numerical computation method has been successfully used to analyze the crisis spreading in the shareholding networks and its controllability when the networks meet random failures or intentional attacks. The main conclusions are follows.

The crisis spreads at a rapid speed and the total Market Capitalization has obviously decreased when the networks meet intentional attack. It means that the intentional attack on hub vertices produces quite a lot of damage. The overall trends of crisis spreading in the networks can be viewed through the changes of the number of vertices in susceptible state, infected state, and removed state. The descent speed of the number of susceptible vertices when networks meet intentional attack is faster than that when networks meet random failure. The rising speed of the number of infected vertices (and removed vertices) when networks meet intentional attack is faster than that when networks meet random failure. Thus, the networks meeting intentional attack show more obviously vulnerability than the networks meeting random attack. It mainly attributes to the enterprises in the sector of Finance and Insurance, and some super-scale companies. The relationships among these enterprises and the other large-scale listed companies or the holding companies are closed. Therefore, intentional attack has huge damage on the shareholding networks. At the same time, these companies are indispensable for our life. Once these companies go bankrupt, the rumor will accelerate the crisis spreading. Then the phenomenon of domino effect may appear easily. Correspondingly, an economic crisis takes place.

Finally, The network-based structural controllability theory conducts controllability research on the network when it is subjected to intentional attacks and random failures. The research shows that if the network needs to be controlled globally, the policy-oriented role and the public opinion control strategy should be used in the crisis. Under normal circumstances, controlling a small part of driver vertices representing listed companies, applying appropriate control strategies, and using its characteristics of high efficiency of sending information can effectively control the stock market. In this way, the government can avoid to get into the financial whirlpool and speed a lot of funds on relieving the initial failure listed companies.

\section{Further Discussion}

As mentioned above, more than $70 \%$ of the Market Capitalization of Chinese stock markets has vaporized during the year of 2008. At the same period, the maximum falling range of Hang Seng Index is 65\% during the year of 2007 and 2008, where the total Market Capitalization of A-shares issued by some state-owned listed companies including 600019, 600028, 601088, 601857, 601628, 601318, 601600, 601988, 
601601, 601919, and 601111 has vaporized ten trillion CNY since the end of 2007. Why did so serious vaporized phenomenon of state-owned enterprises appear? Now, we need to recall that the purpose of founding the Chinese mainland stock markets is to solve the financing difficulties of stateowned enterprises. The Market Capitalization of Chinese mainland stock markets is 26 trillion CNY on December 2011, while the Market Capitalization of state-owned enterprises is 20.3 trillion CNY. Thus, the fluctuation trends of Chinese mainland stock markets are under the influence of stateowned enterprises deeply.

Hong Kong stock markets are developed stock markets and they have a relatively better risk resistance capacity than mainland stock markets because of highly opening level and reasonable economic structure. Just as Justin Yifu Lin (former Chief Economist and Senior Vice President of the World Bank.) points that many problems in Chinese financial system are caused by the problem of state-owned enterprises, we also believed that the poor risk resistance capacity of Chinese financial system and the current low levels of Chinese mainland stock markets mainly attribute to the numerous large-scale listed companies and large-scale holding companies in Chinese stock markets.

Perhaps only when we solve the problems left over by history about state-owned enterprises, can we improve the risk resistance capacity of Chinese financial system. The initial thought of this paper is to establish a crisis spreading model to find out effective strategies for controlling the widely spreading crises in stock markets and to come up with some policy suggestions for the healthy development of Chinese stock markets. It is a pity that the current model established in this paper cannot simulate the crisis spreading in Chinese stock markets accurately. Due to some variables of the model needing further confirmation and many realistic circumstances needing consideration, such as the investors' sentiment and investment behavior, the public opinions, and some other macroeconomic factors, this model is only a simplified academic model.

However, it is worth celebrating that this method discussed in this paper gives us a possible way yet to explore the crisis speeding mechanism and its controllability. In addition, the SIR model proposed in this paper is also beneficial to master the transmission mechanism of crisis spreading on the stock markets and decrease the loss of the economic entity. Further research on the SIR model can help to reveal the dissemination process and root cause of financial crisis.

\section{Data Availability}

The data in text format or EXCEL format used in our manuscript to support the findings of this study are extracted from the RESSET Financial Research Database (http://www.resset.cn/), including all the stocks issued in the Shanghai Stock Exchange and the Shenzhen Stock Exchange before December 31, 2009. The documents we selected include the Main Stockholders List and Ownership Structure and the Yearly Market Capitalization (CNY) of all listed companies. The database was supplied by Beijing Gildata Resset Data Tech Co., Ltd., under license and so cannot be made freely available. Requests for access to these data should be made to Beijing Gildata Resset Data Tech Co., Ltd. (address: B502-503, Caizhi Building, Zhongguancun East Road No. 18, Haidian District, Beijing, China. Tel: +86-1082601461).

\section{Conflicts of Interest}

The authors declare that they have no conflicts of interest.

\section{Acknowledgments}

This research is supported by the National Natural Science Foundation of China (No. 71701036), MOE Project of Humanities and Social Sciences (No. 15YJC790072), the Social Science Fund in Hebei province (No. HB14YJ098), and the Fundamental Research Funds for the Central Universities (N162301001).

\section{References}

[1] H. W. Hethcote, “The mathematics of infectious diseases," SIAM Review, vol. 42, no. 4, pp. 599-653, 2000.

[2] R. Pastor-Satorras and A. Vespignani, "Epidemic spreading in scale-free networks," Physical Review Letters, vol. 86, no. 14, pp. 3200-3203, 2001.

[3] A. Ramani, A. S. Carstea, R. Willox, and B. Grammaticos, "Oscillating epidemics: a discrete-time model," Physica A: Statistical Mechanics and its Applications, vol. 333, no. 1-4, pp. 278-292, 2004.

[4] C. Piccardi and R. Casagrandi, "Inefficient epidemic spreading in scale-free networks," Physical Review E: Statistical, Nonlinear, and Soft Matter Physics, vol. 77, no. 2, 2008.

[5] Y. Moreno, R. Pastor-Satorras, and A. Vespignani, "Epidemic outbreaks in complex heterogeneous networks," The European Physical Journal B, vol. 26, no. 4, pp. 521-529, 2002.

[6] Y. Moreno, J. B. Gómez, and A. F. Pacheco, "Epidemic incidence in correlated complex networks," Physical Review E: Statistical, Nonlinear, and Soft Matter Physics, vol. 68, no. 3, 2003.

[7] Y. Moreno, M. Nekovee, and A. F. Pacheco, "Dynamics of rumor spreading in complex networks," Physical Review E: Statistical, Nonlinear, and Soft Matter Physics, vol. 69, no. 6, Article ID 066130, 2004.

[8] Y.-Y. Liu, J.-J. Slotine, and A.-L. Barabási, "Controllability of complex networks," Nature, vol. 473, no. 7346, pp. 167-173, 2011.

[9] T. Jia and A. Barabási, "Control Capacity and A Random Sampling Method in Exploring Controllability of Complex Networks," Scientific Reports, vol. 3, no. 1, 2013.

[10] T. Jia, Y. Liu, E. Csóka, M. Pósfai, J. Slotine, and A. Barabási, "Emergence of bimodality in controlling complex networks," Nature Communications, vol. 4, no. 1, 2013.

[11] Z. Z. Yuan, C. Zhao, Z. R. Di, W.-X. Wang, and Y.-C. Lai, "Exact controllability of complex networks," Nature Communications, vol. 4, 2013.

[12] J. Sun and A. E. Motter, "Controllability Transition and Nonlocality in Network Control," Physical Review Letters, vol. 110, no. 20, 2013.

[13] Y.-Y. Ma, X.-T. Zhuang, and L.-X. Li, "Research on the relationships of the domestic mutual investment of China based on the cross-shareholding networks of the listed companies," Physica 
A: Statistical Mechanics and its Applications, vol. 390, no. 4, pp. 749-759, 2011.

[14] J. A. Bondy and U. S. R. Murty, Graph Theory with Applications, Macmillan Press, New York, NY, USA, 1976.

[15] "Rules Governing the Listing of Stocks on Shanghai StockExchange," http://english.sse.com.cn/laws/framework/c/4547752 .pdf. (Revised in 2018).

[16] R. Albert and A. L. Barabási, "Statistical mechanics of complex networks," Reviews of Modern Physics, vol. 74, no. 1, pp. 47-97, 2002.

[17] Y. Ma and L. Li, "Research on the topology, community and robustness of the correlated networks of listed companies, funds and their main holders in China," in Proceedings of the 2012 International Conference on Information Management, Innovation Management and Industrial Engineering, ICIII 2012, pp. 40-46, China, October 2012.

[18] A. L. Barabasi and R. Albert, "Emergence of scaling in random networks," Science, vol. 286, pp. 509-512, 1999.

[19] M. Zweig, Martin Zweig's Winning on Wall Street, Grand Central Publishing, 1997.

[20] C. Moore, G. Ghoshal, and M. E. J. Newman, "Exact solutions for models of evolving networks with addition and deletion of nodes," Physical Review E: Statistical, Nonlinear, and Soft Matter Physics, vol. 74, no. 3, Article ID 036121, 2006.

[21] C. Piccardi, A. Colombo, and R. Casagrandi, "Connectivity interplays with age in shaping contagion over networks with vital dynamics," Physical Review E: Statistical, Nonlinear, and Soft Matter Physics, vol. 91, no. 2, 2015.

[22] R. Cohen, S. Havlin, and D. Ben-Avraham, "Efficient immunization strategies for computer networks and populations," Physical Review Letters, vol. 91, no. 24, Article ID 247901, 2003.

[23] A. Barrat, M. Barthélemy, and A. Vespignani, Dynamical Processes on Complex Networks, Cambridge University Press, Cambridge, UK, 2008. 


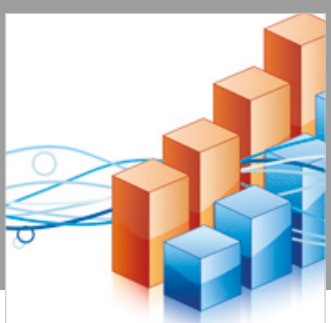

Advances in

Operations Research

\section{-n-m}
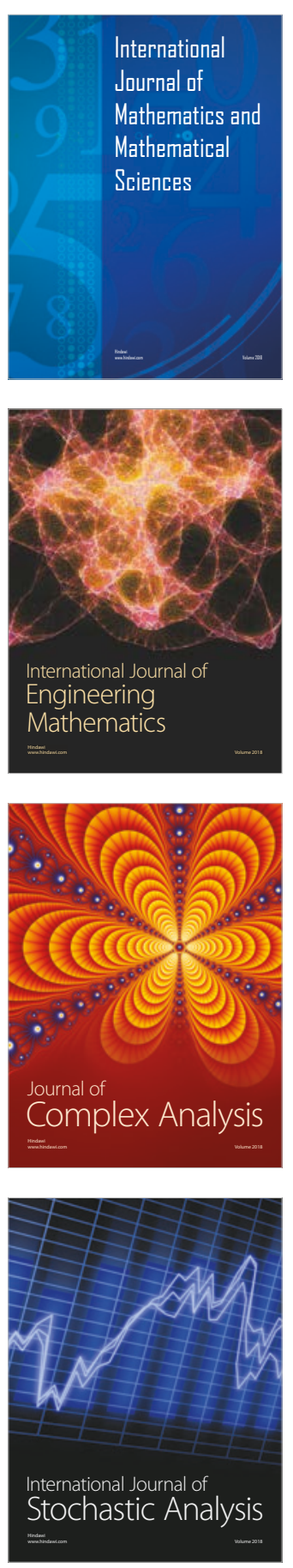
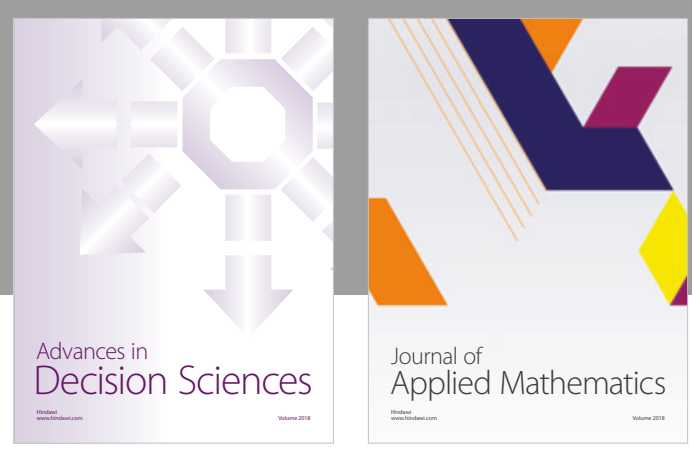

Journal of

Applied Mathematics
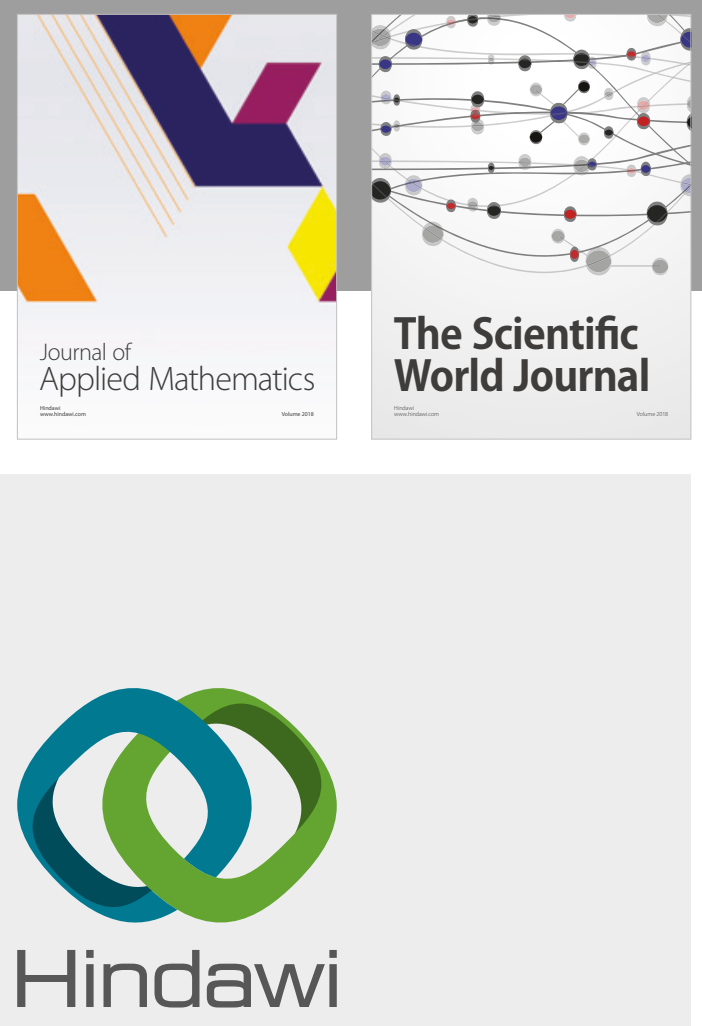

Submit your manuscripts at

www.hindawi.com

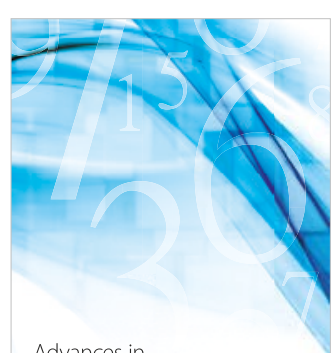

Advances in
Numerical Analysis
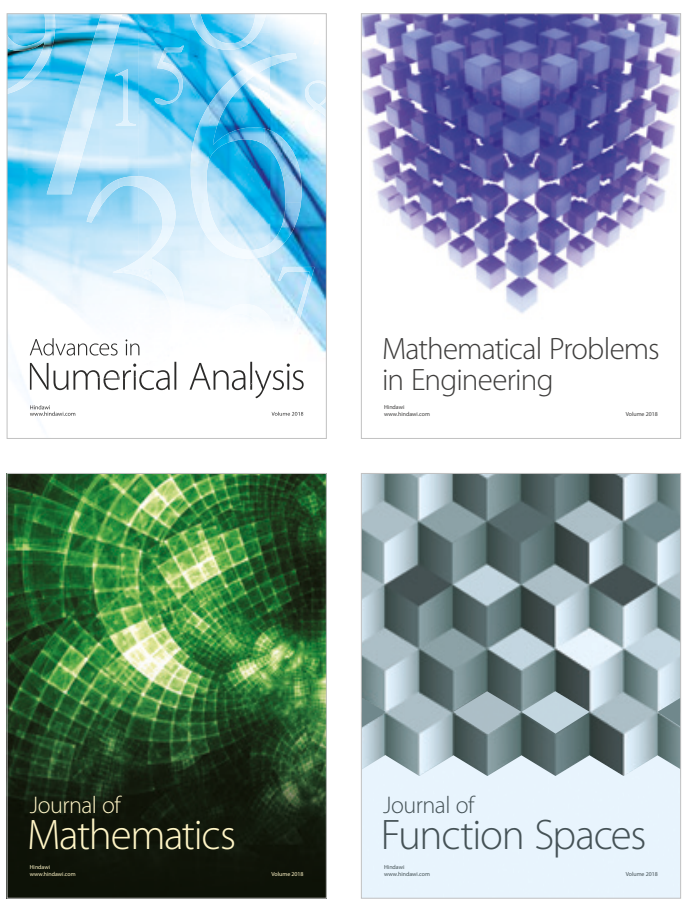

Mathematical Problems in Engineering

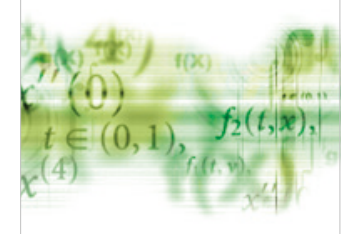

International Journal of

Differential Equations

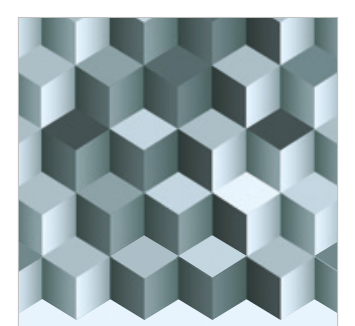

Journal of

Function Spaces
The Scientific

World Journal

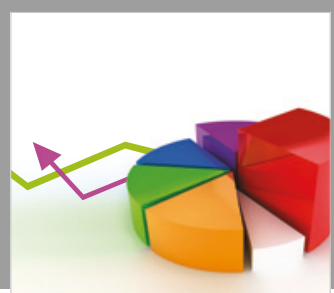

Journal of

Probability and Statistics
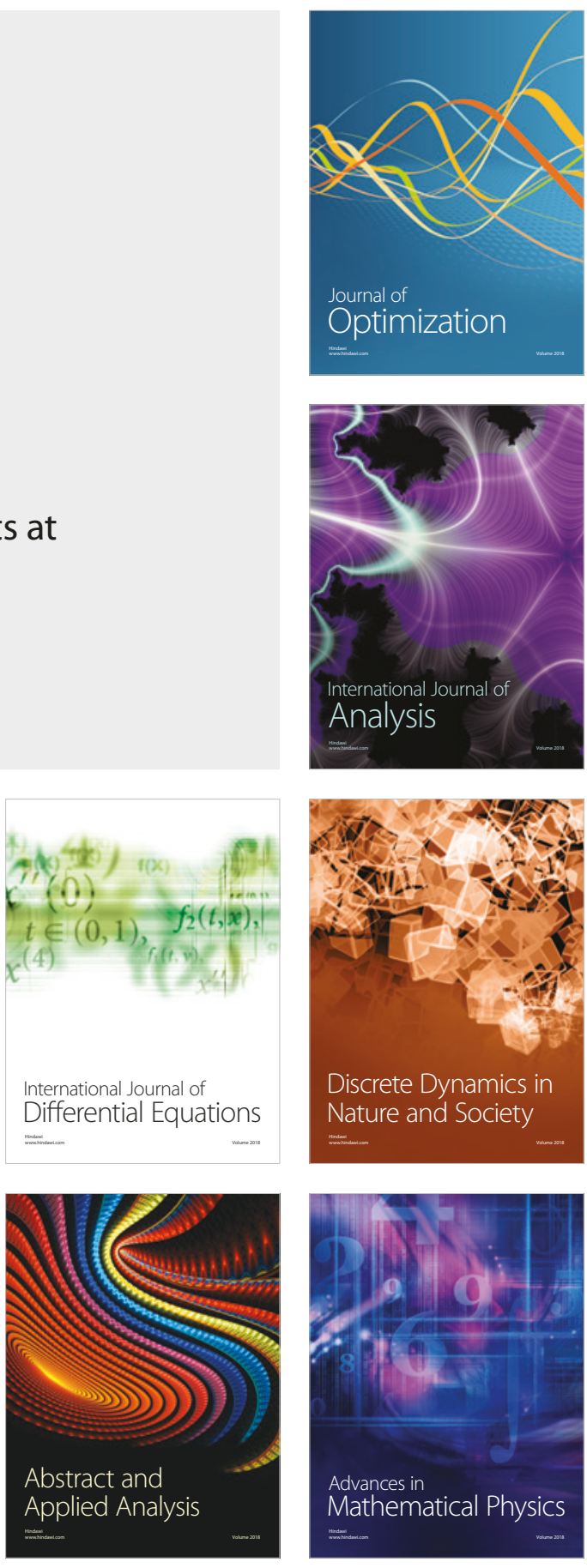